\title{
A Fast QR-RLS algorithm based on Givens Rotation for DPD application
}

\author{
Huadong Wang ${ }^{1 \mathrm{a})}$, Hong Ma ${ }^{1}$, Jinfeng Chen ${ }^{1}$, and Wei Xue ${ }^{2}$ \\ ${ }^{1}$ Department of Electronics and Information Engineering, Huazhong university of \\ science and technology, Wuhan 430074, China \\ ${ }^{2}$ Mechanical Electronic Information Institution, China University of Geosciences, \\ Wuhan 430074, China \\ a)wanghuadong1977@hotmail.com
}

Abstract: In this paper, a fast model identification algorithm based on Givens rotation is presented for the digital predistortion (DPD) application. Because most of the computation work is operated in the hardware, the new algorithm has much faster identification speed than traditional recursive least square (RLS) method or least square (LS) method. Compared with common fast identification algorithms, the new algorithms exhibits the higher identification precision. And final experiment shows that the adjacent channel power ratio (ACPR) after the DPD with the new algorithm is $-50 \mathrm{~dB}, 8 \mathrm{~dB}$ better than that with the conjugate gradient $(\mathrm{CG})$ method, which verifies that the new algorithm has very good performance on both identification precision and identification speed.

Keywords: digital predistortion, QR-RLS, Givens rotation

Classification: Wireless Communication Technologies

\section{References}

[1] J. C. Pedro and S. A. Maas, "A comparative overview of microwave of microwave and wireless power-amplifier behavioral modeling approaches," IEEE Trans. Microw. Theory Tech., vol. 53, no. 4, pp. 1150-1163, April 2005.

[2] M. Isaksson, D. Wisell, and D. Ronnow, "A Comparative Analysis of Behavioral Models for RF Power Amplifiers," IEEE Trans. Microw. Theory Tech., vol. 54, no. 1, pp. 348-358, Jan. 2006.

[3] D. Morgan, Z. Ma, J. Kim, M. Zierdt, and J. Pastalan, "A generalized memory polynomial model for digital predistortion of RF power amplifiers," IEEE Trans. Signal Process., vol. 54, no. 10, pp. 3852-3860, Oct. 2006.

[4] M. Younes, O. Hammi, and A. Kwan, "An Accurate Complexity-Reduced PLUME Model for Behavioral Modeling and Digital Predistortion of RF Power Amplifiers," IEEE Trans. Ind. Electron., vol. 58, no. 4, pp. 13971405, April 2011.

[5] X. D. Zhang, Matrix analysis and application, Tsinghua University Press, Beijing, 2004. 
[6] Y. J. Seong, C. S. Cho, and J. W. Lee, "Digital pre-distortion architecture for RF power amplifier based on affine projection algorithm," Electronics letters., vol. 48, no. 15, pp. 947-948, July 2012.

[7] H. Jiang, X. Yu, and P. A. Wilford, "Digital Predistortion Using Stochastic Conjugate Gradient Method," IEEE Trans. Broadcast., vol. 58, no. 1, pp. 114-123, March 2012.

[8] L. Ding, G. T. Zhou, D. R. Morgan, Z. Ma, and J. S. Kenney, "A robust digital baseband predistorter constructed using memory polynomial," IEEE Trans. Commun., vol. 52, no. 1, pp. 159-165, Jan. 2004.

[9] D. G. Manolakis, V. K. Ingle, and S. M. Kogon, Statistical and Adaptive Signal Processing, Artech House, Norwood MA, 2005.

\section{Introduction}

The digital predistortion technology has been widely used in wireless communication system to improve the linearity of the transmitter and many researches have been done during last decade. Most of early works are focused on the nonlinear model of the power amplifier (PA) and the DPD identification algorithm has seldom been discussed because the identification of these models is very simple $[1,2]$. However, according to the increase of the signal bandwidth, more and more complex models are used to describe the nonlinearity characteristics of the wide-band power amplifier [3, 4], which means that more coefficients are required in the model expression for performance enhancement and the longer time is needed during the model identification process. It makes the identification algorithm become one of current research focuses in the DPD field.

Among those widely used adaptive algorithms [5], the least square (LS) method shows best performance than other algorithms, but it has very high calculation complexity and needs lots of random access memory (RAM) resource during the calculation process. The recursive least square (RLS) method has similar performance as the LS method but it needs inverse matrix operation during the calculation process. On the contrary, the normalized least mean square (NLMS) method has the least calculation complexity than other methods but the worst performance and the slowest convergence speed. To make a tradeoff between RLS method and NLMS method, some new algorithms such as CG and affine projection (AP) are presented and it seems that these algorithms exhibit middle performance on both precision and speed $[6,7]$.

In this letter, a new algorithm called QR-RLS based on Givens Rotation (QR-RLSGR) is introduced. In this algorithm, the input signal is disposed in series like the RLS method, the Givens rotation is used to convert the matrix equation in the identification process to upper triangle matrix, and Gauss elimination method is used to solve final triangle matrix equation. Compared with traditional RLS method, it exhibits many advantages. Firstly, the matrix inverse operation can be gotten rid of from the identification process. Secondly, the new algorithm needs far smaller RAM resource during identi- 
fication process than the LS algorithm. Thirdly, the Givens rotation can be implemented in the hardware by using Cordic processor and in turn greatly improve the computation speed of the algorithm. To evaluate the precision of the new algorithm, the normalized error of the QRRLSGR algorithm is compared with that of other common algorithms, and finally, it is applied to an actual DPD system to test its potential on the linearity enhancement of the power amplifier.

\section{The model expression of DPD system}

The principle of adaptive DPD system is presented in [8], in which the inverse model of the power amplifier is identified by the input signal and the output signal of the power amplifier, and is sent to the predistortion (PD) module. The expression of the inverse model can be written as following.

$$
y(n)=f\left(a_{i}, X(n)\right)
$$

in which

$$
X(n)=\left[x(n), \ldots x\left(n-M_{c}\right)\right]
$$

where $x(n)$ is the feedback signal, $y(n)$ is the input signal, $M_{c}$ is the memory depth of the model, and $a_{i}$ is the model coefficient.

For the most behavioral models based on polynomial structure, $a_{i}$ can be identified by solving following matrix equation since Equation (1) is a linear equation for $a_{i}$.

$$
Y=X A
$$

where $X$ is input parameter matrix, $Y$ is output vector, $A$ is coefficient vector, and $M$ is the number of the model coefficients.

$$
\begin{gathered}
Y=[y(n), \ldots, y(1)]^{T} \\
X=\left[X_{n}, \ldots, X_{1}\right]^{T} \\
A=\left[a_{1}, \ldots, a_{2}, \ldots, a_{M}\right]^{T} \\
X_{n}=\left[x_{1}(n), x_{2}(n), \ldots, x_{M}\right]^{T}
\end{gathered}
$$

\section{QR-RLS based on Givens rotation}

For a $N \times M$ matrix $(N>M)$, the Givens rotation can be used to eliminate any element in the matrix as shown in (8) [9]. And after $M(N-2)+1$ Givens rotations, all matrix elements below diagonal are zero and the matrix becomes a upper triangle matrix.

$$
\begin{aligned}
& {\left[\begin{array}{lll}
c_{1} & \mathbf{0}^{H} & s_{1}^{*} \\
\mathbf{0} & \mathbf{I} & \mathbf{0} \\
-s_{1} & \mathbf{0}^{H} & c_{1}
\end{array}\right] \quad\left[\begin{array}{llll}
\sqrt{\lambda} \widetilde{r}_{11}(n-1) & \ldots & \sqrt{\lambda} \widetilde{r}_{1 M}(n-1) & \sqrt{\lambda} \widetilde{k}_{1}(n-1) \\
\mathbf{0} & \ddots & \vdots & \vdots \\
x_{1}(n) & \ldots & x_{M}(n) & y(n)
\end{array}\right]} \\
& =\left[\begin{array}{llll}
\widetilde{r}_{11}(n) & \ldots & \widetilde{r}_{1 M}(n) & \widetilde{k}_{1}(n) \\
\mathbf{0} & \ddots & \vdots & \vdots \\
0 & \ldots & x_{M}^{(2)}(n) & y^{(2)}(n)
\end{array}\right]
\end{aligned}
$$




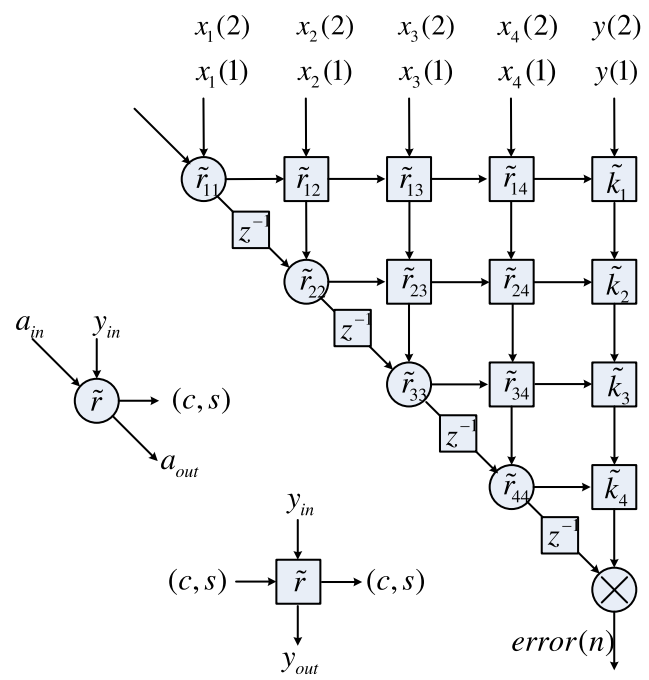

Fig. 1. The implementation of the QR-RLS algorithm by systolic array architecture and functional description of its processing elements [9]

$$
\begin{aligned}
c_{1} & =\frac{\sqrt{\lambda} \widetilde{r}_{11}(n-1)}{\widetilde{r}_{11}(n)} s_{i}=\frac{x_{1}^{*}(n)}{\widetilde{r}_{11}(n)} \\
r_{11}(n) & =\sqrt{\lambda\left|\widetilde{r}_{11}(n-1)\right|^{2}+\left|x_{1}^{*}(n)\right|^{2}}
\end{aligned}
$$

By this method, matrix Equation (3) can be converted to new matrix Equation (11), where $\widetilde{R}$ is upper triangle matrix, and $\widetilde{K}$ is new output vector. The new triangle matrix equation can be solved by Gauss elimination method with very fast speed in the digital signal process (DSP) chip.

$$
\widetilde{R} A=\widetilde{K}
$$

As discussion in the introduction, the Givens rotation operation can be implemented in parallel using CORDICs in the FPGA device, as illustrated in Fig. 1. The arriving new input data $\left[X_{n}^{T} y(n)\right]$ are fed from the top and propagate downward. The Givens rotation parameters are calculated in the boundary cells and propagate from left to right. After all input data are processed, the array performs the matrix $\widetilde{R}$ and the vector $\widetilde{K}$,

\section{Experiment result}

In our experiment, A TD-LTE remote radio unit (RRU) is used as test platform for performance evaluation of the DPD algorithm. It consists of digital intermediate frequency module, transmitter, the 10-W Doherty power amplifier designed using MRF8P26080 LDMOS transistors, and feedback unit with sample rate $184.32 \mathrm{MHz}$, which is used to capture the signal from the output of the PA. The QR-RLSGR algorithm is implemented in the Xilinx FPGA device and Gauss elimination method is implemented in Microblaze processor embedding in the FPGA.

To compare the performance of different algorithms, the QR-RLSGR method, CG method, the LS method and NLMS method have been used to 

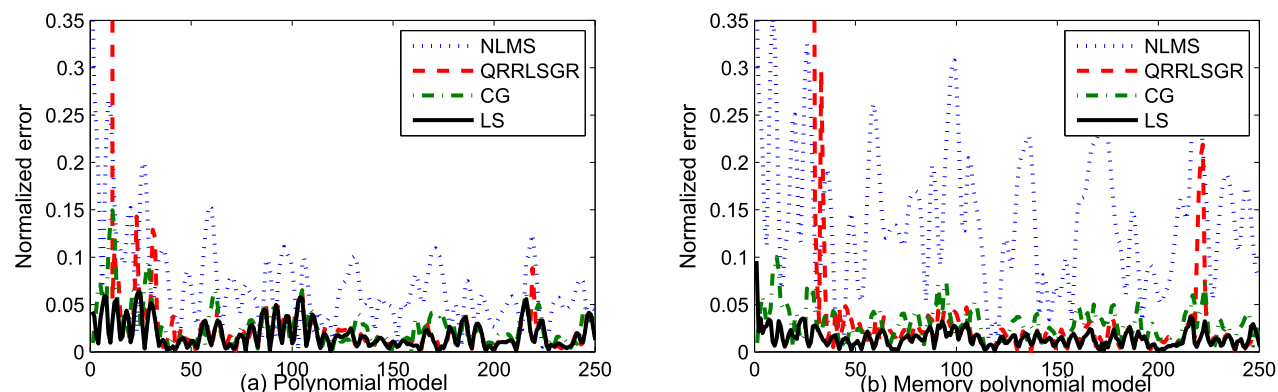

Fig. 2. The normalized model error $e(n)$ with different algorithms during iteration process.( (a) polynomial model with $N_{c}=7$; (b) MP model with $N_{c}=7$ and $M_{c}=3$ )

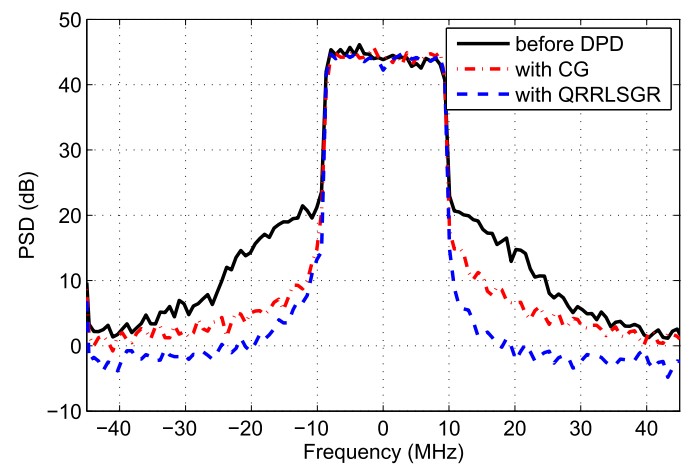

Fig. 3. The output spectrums of the PA before and after the DPD with QR-RLSGR and CG algorithms

identify both of the polynomial model and memory polynomial (MP) model in our system, whose expression is given in (13) and (14), where $N_{c}$ is the nonlinear order.

$$
\begin{gathered}
y_{\text {poly }}(n)=\sum_{k=1}^{N_{c}} a_{k} x(n)|x(n)|^{k-1} \\
y_{\mathrm{MP}}(n)=\sum_{m=0}^{M_{c}} \sum_{k=1}^{N_{c}} a_{m, k} x(n-m)|x(n-m)|^{k-1}
\end{gathered}
$$

In Fig. 2, the normalized errors of the QRRLSGR method are smaller than that of either the NLMS algorithm or the CG algorithm on both cases, and converge to the normalized errors of the LS method after several iterations. However, the NLMS algorithm exhibits different performances at two cases. It works normally when it is used to the identification of the the polynomial model, but encounters the convergence problem when used to the identification of the MP model.

The reason of this phenomenon might be owned to the vulnerability of the NLMS algorithms to the random noise as many coefficients of the MP model are far smaller than the coefficients of the polynomial model. And because the CG algorithm isn't so sensitive to noise as the NLMS, it exhibits better performance than the latter. 
Finally, the performance of the DPD system with the QR-RLSGR algorithm is tested. The MP model with $N_{c}=7$ and $M_{c}=3$ is used as predistortion model in our DPD system. The response time of the new algorithm is less than 1 second, far smaller than that of the DPD system with traditional LS or RLS algorithm. The output spectrums before and after DPD are given in the Fig. 3, in which the ACPR of the PA's output after DPD is $-50 \mathrm{~dB}, 20 \mathrm{~dB}$ improvement compared with that before DPD. As a comparator, the CG algorithm is also used to identify the DPD model in the experiment, and the ACPR improvement of the DPD with the CG algorithm is about $12 \mathrm{~dB}, 8 \mathrm{~dB}$ lower than that of the DPD with QRRLSGR algorithm. This result illustrates that the new algorithm exhibits a better identification precision than common gradient algorithms, and at the same time greatly enhances the response speed of the DPD system.

\section{Conclusion}

In this paper, a new algorithm called QR-RLSGR is represented. By implying matrix operation in the hardware, the new algorithm can enhance the computation speed greatly, and at the same time keep the high identification precision. The experiment result shows that new algorithm exhibits very good performance on both of the response speed and identification precision. Clearly, the new DPD algorithm has extensive application prospect and is especially suitable for the DPD system with high requirement on both the response time and identification precision. 